\title{
Breakfast glycaemic index and exercise: combined effects on adolescents' cognition
}

Simon B. Cooper ${ }^{\text {a }}$, Stephan Bandelow ${ }^{\text {b }}$, Maria L. Nute ${ }^{\text {b }}$, John G. Morris $^{\text {a }} \&$ Mary E. Nevill ${ }^{\text {a }}$

${ }^{a}$ Sport, Health \& Performance Enhancement (SHAPE) Research Group, Sport Science Department, School of Science and Technology, Nottingham Trent University, Nottingham, NG11 8NS

${ }^{\mathrm{b}}$ Institute of Youth Sport, School of Sport, Exercise and Health Sciences, Loughborough University, Leicestershire, LE11 3TU

\section{Corresponding Author:}

Dr Simon Cooper, SHAPE Research Group, Sport Science Department, School of Science and Technology, Nottingham Trent University, Nottingham, NG11 8NS.

Email: Simon.Cooper@ntu.ac.uk

Telephone: 01158488059

Fax: 01158486636

Dr Stephan Bandelow: S.Bandelow@lboro.ac.uk

Maria Nute: M.L.Nute@lboro.ac.uk

Dr John Morris: John.Morris@ @ntu.ac.uk

Professor Mary Nevill: Mary.Nevill@ntu.ac.uk 


\section{Abstract}

The aim of the present study was to examine the combined effects of breakfast glycaemic index (GI) and a mid-morning bout of exercise on adolescents' cognitive function.

Participants were randomly allocated to a high or low GI breakfast group in a mixed research design, where each participant completed two experimental trials (exercise and resting). Forty-two adolescents (12.4 \pm 0.5 years old), undertook a bout of exercise (ten repeats of level one of the multi-stage fitness test; exercise trial) or continued to rest (resting trial) following consumption of either a high or low GI breakfast. A battery of cognitive function tests (visual search test, Stroop test and Sternberg paradigm) was completed $30 \mathrm{~min}$ before and $45 \mathrm{~min}$ following the exercise.

Average heart rate during exercise was $170 \pm 15$ beats $\cdot \mathrm{min}^{-1}$. On the complex level of the Stroop test, response times improved across the morning following the low GI breakfast on both the exercise and resting trials, though the improvement was greatest on the exercise trial. However, response times only improved on the resting trial following the high GI breakfast $(\mathrm{p}=0.012)$. On the 5 letter level of the Sternberg paradigm, response times improved across the morning following the low GI breakfast (regardless of exercise) and only on the exercise trial following the high GI breakfast $(\mathrm{p}=0.019)$.

The findings of the present study suggest that the combined effects of breakfast GI and exercise in adolescents depend upon the component of cognitive function examined. A low GI breakfast and mid-morning bout of exercise were individually beneficial for response times on the Sternberg paradigm, whereas they conferred additional benefits for response times on the Stroop test.

Keywords: breakfast glycaemic index; acute exercise; youth; attention; working memory; visual search 


\section{Introduction}

There is consensus in the literature that breakfast consumption, when compared to breakfast omission, is beneficial for cognitive function in adolescents $(7,13,35)$. However, it appears that breakfast composition also has a role to play in determining adolescents' cognitive function, with particular interest surrounding the glycaemic index (GI) of breakfast. GI provides a measure of the quality of a carbohydrate by classifying foods according to their effect on postprandial glycaemia (15). Per gram of carbohydrate, consumption of a high GI food results in a higher peak blood glucose concentration and a greater overall glycaemic response, when compared to low GI foods. The related concept of glycaemic load (GL) refers to both the quality and quantity of the carbohydrate, and is calculated by multiplying the GI by the amount of available carbohydrate, then dividing by 100 (25).

Previous research suggests that a low GI breakfast is beneficial for cognitive function in a range of populations, including young children $(14,17)$ and adults (2). In adolescent populations, the weight of available evidence suggests that a low GI breakfast is the most beneficial for cognitive function, especially later in the morning $(8,20,34)$. In contrast some studies have indicated that a high GI breakfast is most beneficial for adolescents' cognitive function $(19,26)$. However, such studies have often used high and low GI breakfasts which are not matched on key variables such as energy and carbohydrate content, and have measured only limited components of cognitive function. Whilst the exact mechanisms for the beneficial effect of a low GI breakfast on cognitive function is unknown, a recent review suggests this effect may be due to a more stable supply of fuel (glucose) to nerve cells and/or favourable modulation of hormones (primarily insulin and cortisol) and neurotransmitters following a low GI breakfast (21). 
Whilst the nutritional effects on cognitive function in adolescents are well documented, the acute effects of a single bout of exercise are less well known. There is some evidence to suggest that exercise has a beneficial effect on adolescents' cognitive function $(5,18,31,37)$. However, a recent meta-analysis suggests that comparisons between studies are difficult to make due to numerous factors moderating the relationship between exercise and cognition, including exercise duration, exercise intensity and component of cognitive function examined (6). More recently, it has been shown that a 10 minute mid-morning bout of exercise enhanced subsequent cognitive function in an adolescent population, as evidenced by faster responses on a test of working memory $45 \mathrm{~min}$ following exercise (9).

One previous study has examined the combined effects of breakfast and exercise on cognitive function in adults (33). The findings of the study suggest that breakfast consumption alone had a negative impact on performance on two attention demanding tasks (Stroop test and rapid visual information processing (RVIP) task), but that these negative effects were reversed following a mid-morning bout of exercise (33). Whilst these findings are of interest to the present study, they must be interpreted cautiously as a post-breakfast impairment of cognitive function is not reported elsewhere in the literature. Furthermore cognitive function was assessed after participants had consumed a mid-morning snack and an ad libitum lunch, both of which have previously been demonstrated to affect cognitive function $(1,3,16)$. Thus, the exact effects of breakfast, exercise and their interaction are difficult to determine.

In everyday situations breakfast and exercise do not exist as separate entities and it is likely that a young person will consume breakfast and then exercise either before school, at morning break or during a physical education class. Whilst the individual effects of breakfast glycaemic index (GI) and a mid-morning bout of exercise on adolescents' cognitive function are well documented, the combined effects of breakfast and exercise have not been previously examined in young people. Thus, the combined effects of these variables on 
cognitive function in adolescents are of particular interest, not only due to their previously unexplored nature, but also due to the importance of cognitive function for scholastic achievement.

Therefore, the aim of the present study is to examine the combined effects of a high or low GI breakfast and 10 min of mid-morning exercise or continuing to rest on cognitive function in an adolescent population. Based on the literature to date we hypothesise that both a low GI breakfast and mid-morning bout of exercise will enhance cognitive function in adolescents. However, the combined effects of breakfast GI and exercise are novel to this study and thus this aspect of the study is exploratory.

\section{Methodology}

\section{1: Participant Characteristics}

Fifty two adolescents (aged 11 to 13 years) were recruited to participate in the study. However, 10 participants failed to complete the study because they were either absent from school for one of the experimental trials $(\mathrm{n}=7)$ or failed to comply with the dietary control conditions $(\mathrm{n}=3)$. During familiarisation, simple measures of height, body mass and waist circumference were taken. Height was measured using a Leicester Height Measure (Seca, Hamburg, Germany), accurate to $0.1 \mathrm{~cm}$. Body mass was measured using a Seca 770 digital scale (Seca, Hamburg, Germany), accurate to $0.1 \mathrm{~kg}$. These measures allowed the determination of Body Mass Index (BMI), calculated by dividing body mass [kg] by the square of the height $\left[\mathrm{m}^{2}\right]$. Waist circumference was measured at the narrowest point of the torso between the xiphoid process of the sternum and the iliac crest, to the nearest $0.1 \mathrm{~cm}$. For descriptive purposes, the anthropometric characteristics of the participants who completed the 
study $(\mathrm{n}=42)$ and a comparison between the high and low GI breakfast groups are provided in table 1.

(Insert table 1 here)

\section{2: Study Design}

The study was approved by the institutions ethical advisory committee (approval number R14-P9). Participants were recruited from a local secondary school and in accordance with the ethical guidelines of the British Education Research Authority for school based research, school level consent was obtained from head teachers. In addition, written parental informed consent was obtained and a health screen questionnaire completed (covering any medical issues relating to the child) to ensure all participants were in good health. Finally, participants signed an assent form on each day of testing, to indicate they were willing to participate in the study.

Each participant undertook a familiarisation session followed by two experimental trials. During familiarisation, which preceded the first experimental trial by 7 days, the protocol of the study was explained to participants and they were provided with an opportunity to familiarise themselves with the methods involved. Participants were allowed to repeat the cognitive function tests until they felt comfortable with them, to negate any potential learning effects.

The study employed a mixed research design, with participants randomly allocated to a high or low glycaemic index (GI) breakfast group. Within each group, participants completed an exercise and resting trial, in a randomised, order balanced crossover design. The experimental 
(exercise and resting) trials were scheduled 7 days apart and participants reported to school at the usual time. Figure 1 shows the experimental protocol.

\section{(Insert figure 1 here)}

Upon arrival at school, participants rested for $10 \mathrm{~min}$ in a seated position, then a capillary blood sample was taken and the mood questionnaire completed. The protocol commenced as participants started breakfast, which they were given 15 minutes to consume. The monitoring period was selected based upon the period of time after which it is suggested the effects of breakfast GI $(8,19,34)$ and a mid-morning bout of exercise $(6,9)$ on cognitive function will become apparent in young people.

\section{3: Dietary Control}

Participants were asked to consume a meal of their choice the evening before their first experimental trial and repeated this meal for the subsequent trial. Following this meal, participants fasted from $10 \mathrm{pm}$. In order to maintain euhydration, participants were allowed to drink water ad libitum during this time. In addition, participants avoided any unusually vigorous exercise for $24 \mathrm{~h}$ prior to each experimental trial. Prior to each experimental trial a telephone call was made to participants to remind them of this information. Upon arrival at school, participants were asked to indicate if they had followed the above requirements. Participants who had not followed these requirements were removed from the study $(n=3)$.

\section{4: Mood Questionnaire}


The mood questionnaire was a modified version of the 'Activation-Deactivation Check List' (AD ACL) short form, which has previously been shown as both a valid and reliable measure of mood (30). The mood questionnaire was completed upon arrival at school (0 min), and again at 30,60 and 120 min (figure 1). The 20 item questionnaire was split into four components of mood; energy, tiredness, tension and calmness, each having five corresponding adjectives on the questionnaire. The original AD ACL short form was piloted in an adolescent population and subsequently five of the adjectives were changed to ensure suitability for the study population, with the modified version being previously used successfully in a similar study population $(8,9)$. The scores on the adjectives for each component of mood were summed, providing an overall score for each component.

In addition, three visual analogue (VAS) scales were used to provide a measure of participants' hunger, fullness and concentration. The VAS scales consisted of a $10 \mathrm{~cm}$ line from one extreme to the other (i.e. not at all to very), with participants indicating the point on the line that applied to them at that moment in time. The specific questions used were 'how hungry do you feel now?', 'how full does your stomach feel now?', and 'how well do you feel you can concentrate now?'.

\section{5: Cognitive Function Tests}

The battery of cognitive function tests was administered via a laptop computer and lasted approximately $15 \mathrm{~min}$. The battery of tests consisted of the visual search test, the Stroop test and the Sternberg paradigm, described in detail elsewhere (7). This testing battery has previously been used to successfully examine both nutritional $(7,8)$ and exercise-induced (9) effects on cognitive function in an adolescent population. The cognitive function tests were administered at 30 and 120 min (figure 1). 
Written instructions appeared on the screen at the start of each test, which were repeated verbally by an investigator. Each cognitive function test was preceded by 3-6 practice stimuli, where feedback was provided regarding whether the participants' response was correct or not. Data from these practice stimuli were discarded and once the test started no feedback was provided. The variables of interest on all cognitive function tests were the response times of correct responses and the proportion of correct responses made.

\section{6: Capillary Blood Samples}

Capillary blood samples were taken upon arrival at school (0 min), and again at 30, 60 and 120 min (figure 1). Participants' hands were warmed via submersion in warm water to increase capillary blood flow. A Unistik single use lancet (Unistik Extra, 21G gauge, $2.0 \mathrm{~mm}$ depth, Owen Mumford Ltd., UK) was used and the blood collected into two $300 \mu$ EDTA coated microvettes (Sarstedt Ltd., UK). Two $25 \mu 1$ whole blood samples were removed using plain pre-calibrated glass pipettes (Hawksley Ltd., UK), immediately deproteinised in $250 \mu 1$ of $2.5 \%$ ice cooled perchloric acid in $1.5 \mathrm{ml}$ plastic vials and centrifuged at $7000 \mathrm{rpm}$ for 4 minutes (Eppendorph 5415C, Hamburg, Germany). The remaining whole blood was also centrifuged at $7000 \mathrm{rpm}$ for 4 minutes (Eppendorph 5415C, Hamburg, Germany) and the plasma removed and placed into $500 \mu \mathrm{l}$ plastic vials. All samples were frozen at $-20{ }^{\circ} \mathrm{C}$ until analysis.

Blood glucose concentrations were determined using a commercially available kit (GODPAP method, GL 2610, Randox, Ireland) and were read photometrically using a Cecil CE393 digital grating spectrophotometer (Cambridge, UK). Plasma insulin concentrations were determined using an enzyme-linked immuno-sorbent assay (ELISA) (Mercodia Ltd., Sweden). 


\section{7: Breakfast}

Breakfast was provided after the resting measures had been taken (figure 1) and participants had 15 min to consume breakfast. The high and low GI breakfasts both contained $1.5 \mathrm{~g} \cdot \mathrm{kg}^{-1}$ body mass available carbohydrate and were matched for energy, protein and fat content. The breakfast composition for a $50 \mathrm{~kg}$ participant is shown in table 2 .

\section{(Insert table 2 here)}

\section{8: Exercise Protocol}

The exercise performed was a modified version of the Multi-Stage Fitness Test (15). The exercise protocol was 10 minutes in duration and consisted of 10 repetitions of stage one (each consisting of $7 \times 20 \mathrm{~m}$ shuttle runs at $8.0 \mathrm{~km} . \mathrm{h}-1$ ), with a $30 \mathrm{~s}$ rest between each repetition. Prior to the exercise, participants were fitted with a Polar Wearlink heart rate monitor and a Polar S610i watch (Polar, Finland). Immediately following each repetition, if heart rate had reached 190 beats $\mathrm{min}^{-1}$ (approximately $90 \%$ of maximum heart rate in this population), participants were instructed to stop running and to walk for the remainder of the test. The duration of the exercise was chosen so it was sufficiently brief to fit into a normal school morning and the same exercise protocol has previously shown to be beneficial for adolescents' cognitive function (9).

\section{9: Statistical Analysis}


The mood and blood data were analysed using PASW Statistics (Version 18, SPSS Inc., Chicago, Il, USA) via three-way Analysis of Variance (ANOVA) (breakfast by exercise by time) with repeated measures on two factors (exercise and session time). Data are presented as mean $\pm \mathrm{SE}$.

The cognitive function data were analysed using $\mathrm{R}$ (www.r-project.org, version 2.9.1). All analyses were conducted using a four-way ANOVA (breakfast by exercise by session time by test level), with repeated measures on three factors (exercise, session time and test level). Data are presented as mean \pm SE. For all analysis, significance was set as $\mathrm{p}<0.05$.

\section{Results}

\section{1: Exercise}

Of the 42 adolescents who completed the study, 29 (69.0\%) completed all 10 repeats of level one of the MSFT without their heart rate reaching the threshold of 190 beats $\mathrm{min}^{-1}$. Overall, participants ran for $9 \pm 2$ min (mean \pm SD) and whilst running, the average heart rate was 173 \pm 13 beats $\mathrm{min}^{-1}$. The participants whose heart rate reached 190 beats $\cdot \mathrm{min}^{-1}$ continued to walk for the remainder of the exercise, during which their heart rate was $149 \pm 19$ beats $\mathrm{min}^{-1}$. However, the total exercise time (running and walking) for all participants was $10 \mathrm{~min}$, with an overall average heart rate of $170 \pm 15$ beats $\mathrm{min}^{-1}$. There were no differences in the total running time, walking time, running heart rate, walking heart rate or overall heart rate between the high and low GI breakfast groups (all $\mathrm{p}>0.05$ ).

\section{2: $\operatorname{Mood}$}


For each dimension of mood assessed by the modified ADACL, there was no difference in the pattern of change across the morning on the exercise and resting trials, between the high and low GI breakfast groups (3-way breakfast by exercise by time interactions, all $\mathrm{p}>0.05$ ). Furthermore, when considering the high and low GI breakfast groups separately, there was no effect of exercise on any components of mood (2-way exercise by time interactions, all $\mathrm{p}>$ 0.05). However, there was a significant effect of the time of morning on each component of mood, with energy increasing following breakfast consumption (from 0-30 min), whereas tiredness, tension and calmness all decreased during this time, before all components of mood stabilised during the 30-120 min monitoring period (main effects of time; energy: $F=17.42$, $\mathrm{p}<0.001$; tiredness: $\mathrm{F}=22.81, \mathrm{p}<0.001$; tension: $\mathrm{F}=7.38, \mathrm{p}=0.001$; calmness: $\mathrm{F}=14.87$, $\mathrm{p}<0.001)$.

Similar to mood, there was no difference in the pattern of change across the morning on the exercise and resting trials, between the high and low GI breakfast groups for hunger, fullness and concentration, as assessed by the VAS scales (3-way breakfast by exercise by time interactions, all $\mathrm{p}>0.05)$. Also, when considering the high and low GI breakfast groups separately, there was no effect of exercise on hunger, fullness or concentration in either the high or low GI breakfast groups (2-way exercise by time interactions, all p > 0.05). However, there was a main effect of the time of morning on each dimension of the VAS scales, with hunger decreasing following breakfast consumption (from 0 to $30 \mathrm{~min}$ ), whereas fullness and concentration increased during this time (main effects of time; hunger: $F=17.36, p<0.001$; fullness: $F=83.74, p<0.001$; concentration: $F=30.01, p<0.001)$.

\section{3: Cognitive Function Tests}


For all timed cognitive tests, the response times were first log-transformed to normalise the distributions, which exhibited the right-hand skew typical of human response times. Minimum response time cut-offs were chosen based on what may reasonably be expected to be the fastest possible human response to the given stimuli (100-300 ms depending on task complexity) to exclude unreasonably fast responses, which relate to key presses before stimuli have even been perceived. Maximum response time cut-offs were determined so as to remove unreasonably long right-hand tails for a normal distribution. All cognitive data are represented as changes across the morning, given that there were no differences in response times or accuracy at time point 1 between trials on any of the cognitive function tests (all $p>$ 0.05). Furthermore, order effects did not affect performance on any of the cognitive function tests (breakfast by exercise by session time by order interactions, all $\mathrm{p}>0.05$ ).

\subsection{1: Visual Search Test}

Response Times: Only response times of correct responses were used for analysis. Using the methods previously described, a minimum response time cut-off of $300 \mathrm{~ms}$ for both test levels and a maximum response time cut-off of $1500 \mathrm{~ms}$ for the baseline level and $10000 \mathrm{~ms}$ for the complex level was set. There was no main effect of breakfast $(F=1.41, p=0.322)$ or exercise $(F=0.27, p=0.611)$ on response times on the visual search test. The effect of the mid-morning bout of exercise on response times was not different between the high and low GI breakfast groups or between the baseline and complex levels of the visual search test (4way breakfast by exercise by session time by test level interaction, $F=1.68, \mathrm{p}=0.200$ ). Furthermore, when analysing the data from the baseline and complex levels separately, the three way (breakfast by exercise by session time) interactions were not significant (baseline, $\mathrm{F}=1.48, \mathrm{p}=0.206$; complex, $\mathrm{F}=1.45, \mathrm{p}=0.229$ ), suggesting that breakfast GI and a mid- 
morning bout of exercise did not combine to affect response times on either level of the visual search test.

Accuracy: There was no main effect of breakfast $(\mathrm{F}=0.55, \mathrm{p}=0.464)$ or exercise $(\mathrm{F}=0.61$, $\mathrm{p}=0.440)$ on accuracy on the visual search test. The effect of the mid-morning bout of exercise on accuracy was not different between the high and low GI breakfast groups or between the baseline and complex levels of the visual search test (4-way breakfast by exercise by session time by test level interaction, $F=0.06, p=0.806$ ). Similarly, when analysing the baseline and complex level data separately, there was no difference in accuracy across the morning between the high and low GI breakfast groups on the exercise and resting trials (3-way breakfast by exercise by session time interactions, baseline level: $F=0.05, p=$ 0.828; complex level: $\mathrm{F}=0.09, \mathrm{p}=0.760$ ). Therefore, overall breakfast GI and a midmorning bout of exercise did not affect accuracy on the visual search test.

\subsection{2: Stroop Test}

Response Times: Only response times of correct responses were used for analysis. Using the methods previously described, a minimum response time cut-off of $250 \mathrm{~ms}$ for both test levels and a maximum response time cut-off of $2500 \mathrm{~ms}$ for the baseline level and $4000 \mathrm{~ms}$ for the complex level was set. There was no main effect of breakfast $(F=3.72, p=0.065)$ or exercise $(\mathrm{F}=2.22, \mathrm{p}=0.136)$ on response times on the Stroop test. However, there was a tendency for the effect of the mid-morning bout of exercise on response times to be different between the high and low GI breakfast groups or between the baseline and complex levels of 
the Stroop test (4-way breakfast by exercise by session time by test level interaction, $\mathrm{F}=$ $3.71, \mathrm{p}=0.054)$.

Upon further analysis of the baseline level, the pattern of change in response times across the morning was the same between the high and low GI breakfast groups and between the exercise and resting trials ( 3 -way breakfast by exercise by session time interaction, $F=0.15$, $\mathrm{p}=0.698$ ). However, on the complex level, there was a significant three-way interaction (breakfast by exercise by session time interaction, $F=6.40, p=0.012$, figure 2 ), in that the greatest improvement in response times was seen on the exercise trial following the low GI breakfast (145 $\pm 35 \mathrm{~ms}$ improvement), followed by similar improvements across the morning on the resting trials (low GI and resting: $55 \pm 35$ ms improvement; high GI and resting: $80 \pm$ $26 \mathrm{~ms}$ improvement), whereas response times were unchanged across the morning on the exercise trial following the high GI breakfast ( $5 \pm 21 \mathrm{~ms}$ improvement). Therefore, overall the low GI breakfast followed by a mid-morning bout of exercise was the most beneficial for response times on the Stroop test.

(Insert figure 2)

Accuracy: There was no main effect of breakfast $(\mathrm{F}=0.11, \mathrm{p}=0.738)$ or exercise $(\mathrm{F}=2.54$, $p=0.111$ ) on accuracy on the Stroop test. The effect of the mid-morning bout of exercise on accuracy was not different between the high and low GI breakfast groups or between the baseline and complex levels of the Stroop test (4-way breakfast by exercise by session time by test level interaction, $F=0.16, p=0.689)$. Similarly, when analysing the baseline and complex level data separately, there was no difference in accuracy across the morning between the high and low GI breakfast groups on the exercise and resting trials on either test level (3-way breakfast by exercise by session time interactions, baseline level: $F=0.01, p=$ 
0.907; complex level: $\mathrm{F}=0.61, \mathrm{p}=0.433)$. Therefore, overall breakfast GI and a midmorning bout of exercise did not affect accuracy on the Stroop test.

\subsection{3: Sternberg Paradigm}

Response Times: Only response times of correct responses were used for analysis. Using the methods previously described, a minimum response time cut-off of $200 \mathrm{~ms}$ and a maximum response time cut-off of $2000 \mathrm{~ms}$ was set for all test levels. There was no main effect of breakfast $(\mathrm{F}=2.44, \mathrm{p}=0.131)$ or exercise $(\mathrm{F}=1.04, \mathrm{p}=0.317)$ on response times on the Sternberg paradigm. The effect of the mid-morning bout of exercise on response times was not different between the high and low GI breakfast groups or between the different test levels of the Sternberg paradigm (4-way breakfast by exercise by session time by test level interaction, $\mathrm{F}=2.31, \mathrm{p}=0.128)$.

When analysing the test levels individually, on both the one- and three-item levels, the pattern of change in response times across the morning following the high and low GI breakfasts was similar between the exercise and resting trials (3-way breakfast by exercise by session time interactions: one-item level, $\mathrm{F}=0.01, \mathrm{p}=0.928$; three-item level, $\mathrm{F}=1.73, \mathrm{p}=$ 0.189). However, on the five-item level, there was a significant three-way interaction (breakfast by exercise by session time interaction, $F=5.54, p=0.019$, figure 3 ), in that following the low GI breakfast response times improved by a similar magnitude across the morning on both the exercise $(35 \pm 30 \mathrm{~ms}$ improvement $)$ and resting $(50 \pm 33 \mathrm{~ms}$ improvement) trials, whereas following the high GI breakfast response times improved across the morning on the exercise trial $(55 \pm 18 \mathrm{~ms}$ improvement), but remained unchanged across the morning on the resting trial $(5 \pm 24 \mathrm{~ms}$ improvement). Therefore, overall whilst response times improved across the morning following a low GI breakfast regardless of exercise, 
following a high GI breakfast response times only improved across the morning on the exercise trial.

\section{(Insert figure 3)}

Accuracy: There was no main effect of breakfast $(\mathrm{F}=0.54, \mathrm{p}=0.468)$ or exercise $(\mathrm{F}=2.33$, $\mathrm{p}=0.127$ ) on accuracy on the Sternberg paradigm. The effect of the mid-morning bout of exercise on accuracy was not different between the high and low GI breakfast groups or between the different levels of the Sternberg paradigm (4-way breakfast by exercise by session time by test level interaction, $F=1.57, p=0.211$ ). Similarly, when analysing each test level separately, there was no difference in accuracy across the morning between the high and low GI breakfast groups on the exercise and resting trials on any test level (3-way breakfast by exercise by session time interactions, one-item level: $\mathrm{F}=3.78, \mathrm{p}=0.052$; threeitem level: $\mathrm{F}=2.50, \mathrm{p}=0.114$; five-item level: $\mathrm{F}=0.12, \mathrm{p}=0.730$ ). Therefore, overall breakfast GI and a mid-morning bout of exercise did not affect accuracy on the Sternberg paradigm.

\section{4: Capillary Blood Samples}

\subsection{1: Blood Glucose Concentration}

There was no difference in the pattern of change in blood glucose concentrations across the morning between the exercise and resting trials, between the high and low GI breakfast groups (3-way breakfast by exercise by time interaction, $\mathrm{F}=2.61, \mathrm{p}=0.053$, figure 4 ). However, there was a tendency for this interaction to be significant, which upon inspection of figure 4 would appear to be due to an elevation of blood glucose concentration following the 
exercise (at $120 \mathrm{~min}$ ), but this only occurs following the high GI breakfast. Indeed, following the high GI breakfast, whilst blood glucose concentrations were similar at 0,30 and 60 min (all $\mathrm{p}>0.05$ ), blood glucose concentrations were significantly higher following the exercise (at $120 \mathrm{~min}$ ), when compared to the resting trial ( $\mathrm{p}<0.001)$. However, following the low GI breakfast, there was no difference in blood glucose concentrations between the exercise and resting trials at any time point (all $\mathrm{p}>0.05)$.

Furthermore, blood glucose concentrations were higher following consumption of the high GI, compared to the low GI, breakfast (breakfast by time interaction, $\mathrm{F}=5.28, \mathrm{p}=0.002$, figure 4), confirming the high and low GI nature of the breakfasts provided.

\section{(Insert figure 4)}

\subsection{2: Plasma Insulin Concentration}

Analysis revealed a significant three-way (breakfast by exercise by time) interaction for plasma insulin concentrations $(\mathrm{F}=4.18, \mathrm{p}=0.007$, figure 5). Upon inspection of figure 5 , it appears that this is due to an elevation of plasma insulin concentration following the exercise (at $120 \mathrm{~min}$ ), but only following the high GI breakfast. Indeed, following the high GI breakfast, whilst plasma insulin concentrations were similar at 0, 30 and 60 min (all p > 0.05), they were significantly higher following the exercise (at $120 \mathrm{~min}$ ), when compared to the resting trial $(\mathrm{p}=0.003)$. However, following the low GI breakfast, there was no difference in plasma insulin concentrations between the trials at any time point (all $\mathrm{p}>0.05$ ).

Furthermore, plasma insulin concentrations were higher following consumption of the high GI, compared to the low GI, breakfast (breakfast by time interaction, $\mathrm{F}=5.24, \mathrm{p}=0.002$, figure 5). 


\section{(Insert figure 5)}

\section{Discussion}

The main findings of the present study were that following the low GI breakfast response times improved across the morning on the Stroop test and Sternberg paradigm on both the exercise and resting trials. Furthermore, the mid-morning bout of exercise conferred an additional benefit for response times on the Stroop test, following the low GI breakfast. However, there was no effect of breakfast GI or a mid-morning bout of exercise on accuracy across all cognitive function tests, nor did breakfast GI and exercise exert a combined effect on accuracy. In addition, breakfast GI and a mid-morning bout of exercise did not affect performance on the visual search test or mood in the adolescents tested. The data from the capillary blood samples confirm the high and low GI nature of the breakfasts provided. Given the lack of intervention studies examining the combined effects of nutrition and exercise on cognitive function in young people, the findings of the present study are novel and suggest that breakfast composition (GI) and exercise interact to affect cognitive function in an adolescent population.

\section{1: Stroop Test}

The Stroop test is a commonly used measure of selective attention and executive function $(28,32)$. The findings of the present study suggest that response times on the complex level of the Stroop test are enhanced across the morning following the low GI breakfast (on both the exercise and resting trials), which is in line with previous findings (8). However, it has previously been suggested that a high GI breakfast is beneficial for performance on the Stroop test, but only when the breakfast also had a high glycaemic load (GL) (19), whereas 
other studies have reported no effects of breakfast GI or GL on adolescents' performance on the Stroop test (20). However, the studies demonstrating a beneficial effect of a high GI breakfast have tended to suffer from a number of methodological weaknesses, including; providing meals of differing GI/GL which are not matched on other key variables (such as energy and carbohydrate content) and not reporting whether performance on the Stroop test was assessed via response times and/or accuracy $(19,20)$.

The findings of the present study also suggest that the effects of exercise on response times on the Stroop test depended upon the GI of the breakfast. That is, following a low GI breakfast exercise was beneficial for response times on the Stroop test, whereas following the high GI breakfast response times were unchanged across the morning on the exercise trial. Other studies have examined the effects of exercise on various tests of attention, with conflicting findings. For example, response times on a test of attention created in Matlab (designed to assess frontal lobe function, a key area of the brain for selective attention/executive function) were improved following $30 \mathrm{~min}$ cycling (at a heart rate of approximately 130 beats $\mathrm{min}^{-1}$ ) in 7 and 10 year olds (10), and response times (and accuracy) were also enhanced immediately following 20 min treadmill running in adolescents, albeit compared to a control group using a crossover study design (37). In contrast to these findings, it has also been reported that attention (as assessed by the $\mathrm{d} 2$ test of attention) was not affected following a physical education lesson (when compared to following a science lesson) (24), nor was performance on the Stroop test enhanced following a mid-morning bout of exercise in adolescents (9).

The one previous study to examine the combined effects of breakfast and exercise on cognitive function in adults also used the Stroop test in their testing battery (33). Their findings suggested that there was a post-breakfast impairment of accuracy on the Stroop test, reversed following a mid-morning bout of exercise (33). However, the findings of the present 
study and those in previous studies employing an adolescent population $(7,8)$ do not suggest an impairment of Stroop test performance (or performance on any cognitive function tests) post-breakfast. The findings are consistent however in that following breakfast, a midmorning bout of exercise is beneficial for performance on the Stroop test, albeit only following the low GI breakfast in the present study.

Therefore, it appears that factors such as; the age of the participants, the mode of exercise completed, the timing of the cognitive assessment relative to the exercise and the breakfast GI all influence the effects of an acute bout of exercise on subsequent attention. However, the present study is the first to examine the combined effects of breakfast and exercise on attention in young people and advances knowledge in the area by demonstrating that a midmorning bout of exercise may confer additional benefits to those gained following consumption of a low GI breakfast, for the speed of adolescents' responses on the Stroop test.

\section{2: Sternberg Paradigm}

The Sternberg paradigm is a test of working memory (27). In the present study, the findings indicate that performance on the one- and three-letter levels of the Sternberg paradigm was similar across the morning on the exercise and resting trials following both the high and low GI breakfasts. On the five-letter level, performance improved across the morning following the low GI breakfast, regardless of whether exercise had been performed. However, following the high GI breakfast, whilst response times improved across the morning following the mid-morning bout of exercise, they remained similar across the morning on the resting trial (figure 3). 
Previous studies also indicate that a low GI breakfast was beneficial for adolescents' response times on the Sternberg paradigm (8), in accordance with the improvement in response times seen across the morning in the present study following the low GI breakfast, regardless of whether exercise had been performed. These findings are also consistent with previous findings in the literature, which show that the response times on a test of working memory were enhanced following a low GI breakfast, when compared to both a high GI breakfast and breakfast omission, in 9 to 16 year olds (34). Therefore, the findings of the present study add weight to the available evidence suggesting that a low GI breakfast is beneficial for the speed of adolescents' working memory, whilst also demonstrating that this effect is evident regardless of a mid-morning bout of exercise.

However, the mid-morning bout of exercise was beneficial for response times across the morning following a high GI breakfast, whereas response times remained similar across the morning on the resting trial (figure 3). These findings are similar to previous data (9), also suggesting that exercise was beneficial for adolescents' response times on the Sternberg paradigm, a test of working memory.

Overall, the findings of the present study suggest a beneficial effect of a low GI breakfast (regardless of exercise) and a mid-morning bout of exercise (following a high GI breakfast) for response times on the Sternberg paradigm. The present study is the first to examine their combined effects, but does suggest that either a low GI breakfast, or a mid-morning bout of exercise enhance response times on the Sternberg paradigm. However, the findings also suggest that by consuming a low GI breakfast and completing a mid-morning bout of exercise, there is no additional beneficial effect on response times above that achieved by either practice alone. 


\section{3: Visual Search Test}

The findings of the present study indicate that performance on the visual search test (assessing perception and simple response times) was not different across the morning on the exercise and resting trials following the high and low GI breakfasts. These results therefore suggest that breakfast GI and a mid-morning bout of exercise do not combine to affect visual perception (as assessed by the visual search test). The effects of breakfast GI on adolescents' performance on the visual search test have not previously been examined. However, previous findings have demonstrated that a mid-morning bout of exercise causes a speed-accuracy trade-off on the complex level of the visual search test, whereby adolescents responded quicker but also with less accuracy following exercise (9). The findings of the present study extend this previous work and demonstrate that there are no combined effects of breakfast GI and a mid-morning bout of exercise on adolescents' performance on the visual search test.

\section{4: Mechanisms}

The mechanisms mediating the effects of breakfast GI and exercise on cognitive function remain unknown, although several potential mechanisms have been suggested. Specifically, a recent review suggests low GI breakfasts may be beneficial for cognitive function due to a more stable supply of fuel (glucose) to nerve cells and/or favourable modulation of hormones (primarily insulin and cortisol) and neurotransmitters (21). The findings of the present study display a similar glycaemic and insulinaemic response to previous studies (8), suggesting that such responses are beneficial for cognitive function in an adolescent population.

In terms of the mechanisms mediating the effects of exercise, it has been suggested that postexercise improvements in cognitive function may be due to increased cerebral blood flow, thereby enhancing the delivery of glucose and oxygen to neural tissues (22). In addition, 
other mechanisms such as changes in signalling within the brain $(12,29)$ and increases in arousal (4) have been proposed to enhance cognition following exercise in young people. However, the exact mechanisms mediating the effects of exercise on cognition remain unknown, and certainly warrant further investigation, as do the mechanisms mediating the combined effects of breakfast GI and exercise on subsequent cognition.

\section{5: Summary and Future Research Directions}

The main findings of the present study are that the effects of breakfast GI and the midmorning bout of exercise depend on the component of cognitive function being measured. For the Stroop test and Sternberg paradigm, response times improved across the morning following the low GI breakfast regardless of whether exercise was completed. However, following the high GI breakfast, exercise was beneficial for response times on the Sternberg paradigm, whereas response times improved across the morning on the resting trial on the Stroop test. In contrast, following the low GI breakfast, the magnitude of improvement in response times across the morning on the Stroop test was greatest on the exercise trial, suggesting that exercise confers an additional benefit to the consumption of a low GI breakfast. Across the components of cognitive function measured, response times seemed to be affected whilst accuracy was not, and these effects were only evident on the more complex levels of the tests employed, indicative of effects on more complex cognitive functions, whereas simpler tasks can be carried out to a similar level regardless of breakfast composition and exercise, in line with previous findings $(8,9)$.

However, further work is required in this field to further examine the combined effects of breakfast composition and exercise on adolescents' cognitive function. In everyday practice, both breakfast consumption and exercise (either during break times or physical education 
lessons) take place before or during the school morning, but the present study is the first to examine their combined effects. In addition, future work should also aim to examine: whether there is an optimal timing for breakfast and exercise during the morning, the effects of breakfasts with differing macronutrient contents, and the effects of different modes, durations and intensities of exercise on subsequent cognition. 


\section{References}

1. Benton D, Slater O, Donohoe RT. The influence of breakfast and a snack on psychological functioning. Physiol Behav, 2001:74;559-71.

2. Benton D, Ruffin MP, Lassel T, Nabb S, Messaoudi M, Vinoy S, DEsor D, Lang V. The delivery rate of dietary carbohydrates affects cognitive performance in both rats and humans. Psychopharm, 2003:166;86-90.

3. Benton D, Jarvis M. The role of breakfast and a mid-morning snack on the ability of children to concentrate at school. Physiol Behav, 2007:90;382-5.

4. Brisswalter J, Collardeau M, René A. Effects of acute physical exercise characteristocs on cognitive performance. Sports Med, 2002:32;555-66.

5. Budde H, Voelcker-Rehage C, Pietraßyk-Kendziorra S, Ribiero P, Tidow W. Acute coordinative exercise improves attentional performance in adolescents. Neurosci Lett. 2002;441:219-23.

6. Chang YK, Labban JD, Gapin JJ, Etnier JL. The effects of acute exercise on cognitive performance: a meta-analysis. Brain Res, 2012;1453:87-101.

7. Cooper SB, Bandelow S, Nevill ME. Breakfast consumption and cognitive function in adolescent schoolchildren. Physiol Behav, 2011; 103:431-9.

8. Cooper SB, Bandelow S, Nute ML, Morris JG, Nevill ME. Breakfast glycaemic index and cognitive function in adolescent school children. Br J Nutr, 2012a:107;1823-32.

9. Cooper SB, Bandelow S, Nute ML, Morris JG, Nevill ME. The effects of a midmorning bout of exercise on adolescents' cognitive function. Ment Health Phys Act, 2012b:5;183-90.

10. Ellemberg D, St-Louis-Deschênes M. The effect of acute physical exercise on cognitive function during development. Psychol Sport Exerc, 2010:11;122-6.

11. Foster-Powell K, Holt SHA, Brand-Miller JC. International table of glycemic index and glycemic load values: 2002. Am J Clin Nutr, 2002:76;5-56.

12. Hillman CH, Pontifex MB, Raine LB, Castelli DM, Hall EE, Kramer AF. The effect of acue treadmill walking on cognitive control and academic achievement in preadolescent children. Neurosci, 2009:159;1044-54.

13. Hoyland A, Dye L, Lawton CL. A systematic review of the effect of breakfast on the cognitive performance of children and adolescents. Nutr Res Rev, 2009:22;220-43. 
14. Ingwersen J, Defeyter MA, Kennedy DO, Wesnes KA, Scholey AB. A low glycaemic index breakfast cereal preferentially prevents children's cognitive performance from declining throughout the morning. Appetite, 2007:49;240-4.

15. Jenkins DJA, Wolever TMS, Taylor RH, Barker H, Fielden H, Baldwin JM, Bowling AC, Newman HC, Jenkins AL, Goff DV. Glycemic index of foods: a physiological basis for carbohydrate exchange. Am J Clin NUtr, 1981:34;362-66.

16. Kanarek RB, Swinney D. Effects of food snacks on cognitive performance in male college students. Appetite, 1990:14;15-27.

17. Mahoney CR, Taylor HA, Kanarek RB, Samuel P. Effect of breakfast composition on cognitive processes in elementary school children. Phys \& Beh, 2005:85;635-45.

18. McNaughten D, Gabbard C. Physical exercise and immediate mental performance of sixth-grade children. Perc Motor Skills, 1993:77;1155-9.

19. Micha R, Rogers PJ, Nelson M. Glycaemic potency of breakfast predicts cognitive function and mood in adolescent schoolchildren. Proc Nutr Soc, 2008:67;E364.

20. Micha R, Rogers PJ, Nelson M. The glycaemic potency of breakfast and cognitive function in school children. Eur J Clin Nutr, 2010:64;948-57.

21. Philippou E, Constantinou M. The influence of glycaemic index on cognitive functioning: a systematic review of the evidence. Adv in Nutr, 2014:5;119-30.

22. Querido JS, Sheel AW. Regulation of cerebral blood flow during exercise. Sports Med, 37;766-82.

23. Ramsbottom R, Brewer J, Williams C. A progressive shuttle run test to estimate maximal oxygen uptake. Br J Sports Med, 1988:22;141-4.

24. Raviv S, Low M. Influence of physical activity on concentration among junior highschool students. Perc Motor Skills, 1990:70;67-74.

25. Salmeron J, Manson JE, Stampfer MJ, Colditz GA, Wing AL, Willett WC. Dietary fiber, glycemic load, and risk of non-insulin-dependent diabetes mellitus in women. $J$ Am Med Asooc, 1997:277;472-7.

26. Smith MA, Foster JK. The impact of a high versus a low glycaemic index breakfast cereal meal on verbal episodic memory in healthy adolescents. Nutr Neurosci, 2008:11;219-227.

27. Sternberg S. Memory-scanning: mental processes revealed by reaction-time experiments. Am Sci, 1969:57;421-57.

28. Stroop JR. Stroop Test. J Exp Psych, 1935:18;643-62. 
29. Stroth S, Kubesch S, Dieterle K, Ruschow M, Heim R, Kiefer M. Physical fitness, but not acute exercise modulates event-related potential indices for executive control in healthy adolescents. Brain Res, 2009:1269;114-24.

30. Thayer RE. Activation-deactivation checklist: current overview and structural analysis. Psych Report, 1986:58;607-14.

31. Travlos AK. High intensity physical education classes and cognitive performance in eighth grade students: an applied study. Int J Sp Ex Psych, 2010:8;302-11.

32. Van Zomeren AH, Brouwer WH. Assessment of attention. In: Crawford JR, Parker DM, McKinlay WW, editors. A Hanbook of Neuropsychological Assessment. UK: Lawrence Erlbaum Associates Ltd; 1992. p. 241-66.

33. Veasey RC, Gonzalez JT, Kennedy DO, Haskell CF, Stevenson EJ. Breakfast consumption and exercise interact to affect cognitive performance and mood later in the day. A randomized control trial. Appetite, 2013:68;38-44.

34. Wesnes KA, Pincock C, Richardson D, Helm G, Hails S. Breakfast reduces declines in attention and memory over the morning in schoolchildren. Appetite, 2003:41;32931.

35. Widenhorn-Müller K, Hille K, Klenk J, Weiland U. Influence of having breakfast on cognitive performance and mod in 13- to 20-year-old high school students: results of a crossover study. Pediatrics, 2008:122;279-84.

36. Wolever TMS, Jenkins DJA. The use of the glycemic index in predicting the blood glucose response to mixed meals. Am J Clin Nutr, 1981:43;167-72.

37. Zervas Y, Dabis A, Klissouras V. Influence of physical exertion on mental performance with reference to training. Perc Motor Skills, 1991:72;1215-21. 